\title{
OUTCOME OF HEMISPHERECTOMY FOR REFRACTORY EPILEPSY
}

The clinical features, presurgical workup, and postoperative outcome of 39 patients with medically intractable epilepsy who underwent hemispherectomy from 1996 to 2005 are reported from University of Sao Paulo, Brazil. All patients had preoperative hemiparesis and $76 \%$ were developmentally delayed. Seizures, with onset at 3 years, were tonic in $30 \%$, focal motor in $20 \%$, epilepsia partialis continua (18\%), and complex partial in $10 \%$ of patients. Etiologies included Rasmussen encephalitis (30\%), cortical maldevelopment (23\%), porencephaly $(20 \%)$, gliosis $(15 \%)$, Sturge-Weber syndrome $(5 \%)$, tuberous sclerosis $(2.6 \%)$, and tumors $(2.6 \%)$. Post-surgery at a mean age of 7.5 years (range 2 months to 17 years), $90 \%$ of patients had a $90 \%$ reduction in seizure frequency. An acute worsening of hemiparesis and a hemianopia occurred in all patients, and 3 patients died (1 early with edema and herniation, and 2 late with frequent seizures). A good surgical outcome is expected in patients showing unilateral disease on both video-EEG and MRI. In comparing patients with good or poor outcome, no differences were observed in age of epilepsy, age at surgery, time at seizure recurrence, sex, status epilepticus, side of surgery, surgical technique, and seizure etiology. Patients with bilateral or diffuse EEG findings did not have worse outcomes and did not preclude epilepsy surgery. AED therapy was maintained and in reduced doses for at least 2 years in patients with monotherapy; those on polytherapy had treatment modified to monotherapy when possible. (Terra-Bustamante VC, Inuzuka LM, Fernandes RMF et al. Outcome of hemispheric surgeries for refractory epilepsy in pediatric patients. Childs Nerv Syst March 2007;23:321-326). (Respond: Dr HR Machado, Departmento de Neurologia, Centro de Cirurgia de Epilepsia-CIREP, Campus Universitario, Ribeirao Preto-SP, CEP 14048-900, Sao Paulo, Brazil).

COMMENT. The Johns Hopkins extensive series of hemispherectomies, 111 cases from 1975-2001, demonstrates a beneficial response in $86 \%$ (seizures controlled in $65 \%$ and occasional in $21 \%$ ). The outcome was particularly favorable in children with Rasmussen syndrome and vascular disorders. Seizures due to migrational disorders were less responsive. (Kossof EH. Neurology 2003;61:887-890).

In the Sao Paulo study, seizures were associated with cortical maldevelopment in 10 patients; seizure control was $100 \%$ in 7 and $75 \%$ in 2 . In 12 with Rasmussen syndrome, seizure control was $95-100 \%$ in 11 and $75 \%$ in 1 . Contrary to the Hopkins data, outcome was not significantly correlated with seizure etiology.

\section{$\underline{\text { ANTIEPILEPTIC DRUGS }}$}

\section{LAMOTRIGINE AND SUDDEN UNEXPECTED DEATH IN EPILEPSY}

Four consecutive cases of sudden unexpected death in epilepsy (SUDEP), occurring in non-hospitalized patients while treated with lamotrigine (LTG), between 1995 and 2005, are reported from Stavanger University Hospital, Norway. All were female gender, the epilepsy was idiopathic, age range was 16 to 37 years, and LTG was monotherapy. Seizures 